\title{
Tumour Lysis Syndrome and Partial Remission Occurring After Administration of a Test Dose of Obinutuzumab
}

\author{
Mohamad Mustafa, Mohamed B Mohamed, Amjad Hayat \\ Department of Haematology, Galway University Hospital, Galway, Ireland
}

Received: $11 / 10 / 2016$

Accepted: $24 / 10 / 2016$

Published: $10 / 11 / 2016$

How to cite this article: Mustafa M, Mohamed MB, Hayat A. Tumour lysis syndrome and partial remission occurring after administration of a test dose of obinutuzumab. EJCRIM 2016;3: doi:10.12890/2016_000516.

Conflicts of Interests: The Authors declare that there are no competing interests.

This article is licensed under a Commons Attribution Non-Commercial 4.0 License

\section{ABSTRACT}

Chronic lymphocytic leukaemia (CLL) is one of the most common haematological malignancies worldwide, with an increasing prevalence in the elderly population. Obinutuzumab is a type II anti-CD20 monoclonal antibody which showed superiority over rituximab in combination chemotherapy with chlorambucil for the treatment of CLL in the CLL11 trial (NCT01010061) and is becoming part of standard first line treatment for CLL in the elderly based on its potent efficacy and benign safety profile. We report the case of a chemotherapy naive patient who develop tumour lysis syndrome despite appropriate prophylaxis, and had partial remission of her disease after receiving only the initial test dose of obinutuzumab.

\section{LEARNING POINTS}

- Chemotherapy-induced tumour lysis syndrome is a serious complication that can occur after even a small dose of chemotherapy.

- Chronic lymphocytic leukaemia patients with strongly positive CD20 cells can be more sensitive to obinutuzumab chemotherapy.

\section{KEYWORDS}

Chronic lymphocytic leukaemia; obinutuzumab; tumour lysis syndrome.

\section{INTRODUCTION}

Obinutuzumab is a glycoengineered potent type II anti-CD20 monoclonal antibody with enhanced antibody-dependent cellular toxicity and direct cell death compared with rituximab ${ }^{[1]}$.

The CLL11 trial was a phase 3 randomized trial of chlorambucil alone or with either obinutuzumab or rituximab in elderly, unfit patients. Progression-free survival (the primary end point) was 26.7 months for patients receiving obinutuzumab plus chlorambucil versus 16.3 months for those receiving rituximab plus chlorambucil ${ }^{[1]}$. This efficacy is the result of obinutuzumab's ability to significantly enhance antibodydependent cellular cytotoxicity (ADCC), decreased complement-dependent cytotoxicity (CDC) and superior direct killing compared to type I antibodies ${ }^{[2]}$. Its glycoengineered structure results in decreased fucosylation of the Fc region of the antibody, which significantly enhances its affinity for the Fc receptor on effector cells, including NK cells and macrophages ${ }^{[3]}$.

Infusion-related reactions (IRRs) are a common side effect of treatment with obinutuzumab. The frequency of IRR of any grade observed in the CLL11 study was $66 \%$ in the obinutuzumab and chlorambucil arm, with $20 \%$ of IRRs being grade $3-4$. In comparison, only $3 \%$ of patients receiving rituximab developed grade 3-4 IRRs ${ }^{[1]}$. However, to the best of our knowledge, the propensity of obinutuzumab to cause tumour 
lysis syndrome (TLS) has not been systematically examined.

In a randomized phase 2 study of obinutuzumab monotherapy in symptomatic, previously untreated patients with chronic lymphocytic leukaemia (CLL), the incidence of TLS was very low, with only three out of 80 enrolled patients experiencing TLS in the first cycle ${ }^{[4]}$.

We report a case of CLL where an initial $100 \mathrm{mg}$ dose of obinutuzumab resulted in rapid onset of TLS.

\section{CASE REPORT}

is An 88-year-old woman with history of CLL with bulky cervical and axillary lymphadenopathy was admitted electively for first-cycle chemotherapy. She had been diagnosed with CLL 2 years previously after she was referred from her primary healthcare centre with enlarged cervical and axillary lymph nodes; she was otherwise asymptomatic. Her medical history included hypertension and atrial fibrillation and she was on diltiazem and lansoprazole. Her blood tests at initial presentation revealed isolated lymphocytosis $(9 \times 108)$ consistent with CLL on flow cytometry.

Computed tomography imaging showed massive lymphadenopathy from the skull base to below the symphysis pubis, no splenomegaly, and no definite extra-lymph node locations except for a small area of hypoperfusion in the liver, with a diameter of $1.2 \mathrm{~cm}$ which could have represented lymphoma.

Bone marrow biopsy showed relatively diffuse infiltration by small monomorphic

lymphocytes in a non-paratrabecular distribution. Background trilineage haematopoiesis was seen and immunohistochemistry confirmed the cells to be strongly positive for CD20 and CD5. CD23 was weakly expressed and CD10 and cyclin D1 were negative.

A histological section from a lymph node biopsy showed a core of lymphoid tissue without normal lymphoid follicles. The sample showed diffuse proliferation of mildly atypical small lymphoid cells with normal histiocytes scattered between them.

The immunohistochemical profile of the atypical lymphoid cells was positive for CD20 and CD43 and negative for CD5, CD10, bcl6, CD23 and cyclin D1.

The patient was assigned to start treatment with obinutuzumab and chlorambucil. Before receiving the first dose of obinutuzumab (100 mg), she was premedicated with $6 \mathrm{mg}$ intravenous rasburicase and continuous intravenous fluid. However, $10 \mathrm{~h}$ after receiving the obinutuzumab infusion, the patient experienced absence-like seizures and confusion, and became febrile.

Serum creatinine rose to $194 \mathrm{mg} / \mathrm{dl} 10 \mathrm{~h}$ later and the patient became oliguric with a urine output of only $100 \mathrm{ml} / \mathrm{day}$. Serum potassium increased to $7.4 \mathrm{mmol} / \mathrm{l}$, serum lactate dehydrogenase to $979 \mathrm{U} / \mathrm{l}$, phosphate to $3.99 \mathrm{mmol} / \mathrm{l}$ and uric acid to $509 \mathrm{mg} / \mathrm{dl}$. Serum calcium decreased to $1.81 \mathrm{mmol} / \mathrm{l}$. A diagnosis of obinutuzumab-induced TLS was made, and the patient was admitted to the intensive care unit, treated with intravenous hydration, intravenous rasburicase, allopurinol and urine alkalinization.

Electrolyte imbalance was corrected with intravenous calcium gluconate, insulin-dextrose and salbutamol. The patient did not need haemodialysis. Renal function gradually recovered, and serum creatinine and urine output were normal on day 5.

Owing to toxicity, treatment was deferred for 6 weeks and the patient was then switched to rituximab, which therapy was uneventful. Repeat CT scan of the chest, abdomen and pelvis 2 months after the first dose of obinutuzumab showed partial remission and significant lymphadenopathy reduction (Fig. 1).
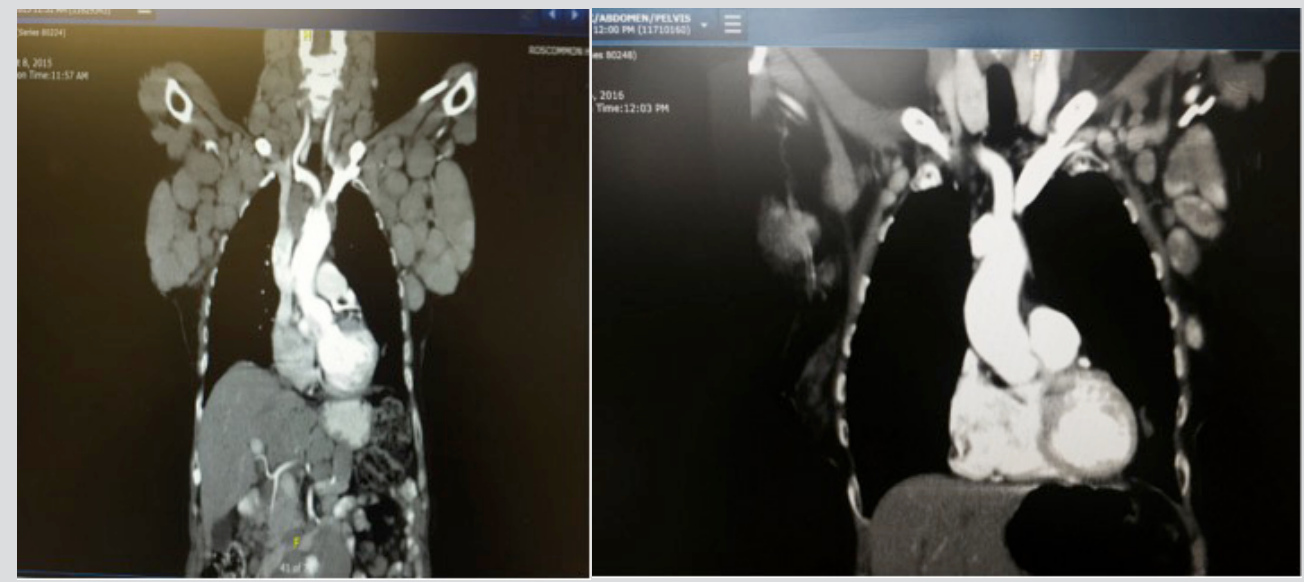

Figure 1 CT scans of the chest, abdomen and pelvis. Left panel: before treatment. Right panel: 6 weeks after treatment 


\section{DISCUSSION}

TLS secondary to chemotherapy has been reported extensively in many types of haematological malignancy, but TLS cases resulting from immunotherapy are less frequently reported. There have been several reports of TLS secondary to rituximab, but according to our best knowledge, very few cases of TLS secondary to obinutuzumab.

Moreover, CLL with WBC <50,000, as in our patient, is considered to have a low risk of TLS, and not to require extensive prophylaxis according to TLS prophylaxis recommendations. Furthermore, the incidence of grade 3 or 4 TLS was $2 \%$ in the obinutuzumab-treated arm of the CLL11 stage II study. Therefore, it seems this case is a rare event.

Our patient exhibited TLS with elevated phosphate, uric acid and lactate dehydrogenase resulting from massive necrosis of lymphoma/ leukaemia cells and release of the intracellular degradation products after administration of an initial $100 \mathrm{mg}$ dose of obinutuzumab despite receiving extensive prophylaxis with rasburicase and continuous hydration. She also had a significant reduction in the size of her mediastinal, hilar and axillary lymph nodes.

The causes of this significant reduction and TLS following a small dose of obinutuzumab remain unclear but may reflect systemic activation of tissue factor as a consequence of rapid antibody-mediated tumour cell depletion. However, it might be that some patients are more sensitive to this type of treatment, especially those who are strongly positive to CD20 like our patient. Previous studies showed patients with higher baseline expression of CD20 on their CLL cells were at greater risk for severe grade early IRR, especially those treated with obinutuzumab. Interestingly, the very rapid tumour reduction occurred in the presence of strongly positive CD20 cells, so whether the obinutuzumab dose should be reduce in such patients awaits further studies.

\section{CONCLUSION}

An obinutuzumab test dose appeared to cause TLS and a significant reduction in lymphadenopathy in a CLL patient with high CD20 expression.

\section{REFERENCES}

1. Goede V, Fischer K, Busch R, Engelke A, Eichhorst B, Wendtner CM, et al. Obinutuzumab plus chlorambucil in patients with CLL and coexisting conditions. N Engl J Med 2014;370:1101-1110.

2. Kern DJ, James BR, Blackwell S, Gassner C, Klein C, Weiner GJ. GA101 induces NK-cell activation and antibody-dependent cellular cytotoxicity more effectively than rituximab when complement is present. Leuk Lymphoma 2014;54:2500-2505.

3. Klein C, Lammens A, Schäfer W, Georges G, Schwaiger M, Mössner E, et al. Epitope interactions of monoclonal antibodies targeting CD20 and their relationship to functional properties. MAbs 2013;5:22-33.

4. Byrd JC, Flynn JM, Kipps TJ, Boxer M, Kolibaba KS, Carlile DJ, et al. Randomized phase 2 study of obinutuzumab monotherapy in symptomatic, previously untreated chronic lymphocytic leukemia. Blood 2016;127:79-86. 